\title{
Polyoxometalate as Control Agent for the Doping in HgSe Self-Doped Nanocrystals
}

\author{
Bertille Martinez ${ }^{1,2}$, Clément Livache ${ }^{1,2}$, Elisa Meriggio ${ }^{1}$, Xiang Zhen $\mathrm{Xu}^{2}$, Hervé Cruguel ${ }^{1}$, \\ Emmanuelle Lacaze ${ }^{1}$, Anna Proust ${ }^{3}$, Sandrine Ithurria ${ }^{2}$, Mathieu G. Silly ${ }^{4}$, Gregory Cabailh ${ }^{1}$, \\ Florence Volatron ${ }^{3}$, Emmanuel Lhuillier ${ }^{1^{*}}$ \\ ${ }^{1}$ Sorbonne Université, CNRS, Institut des NanoSciences de Paris, INSP, F-75005 Paris, France. \\ ${ }^{2}$ Laboratoire de Physique et d'Etude des Matériaux, ESPCI-Paris, PSL Research University, \\ Sorbonne Université Univ Paris 06, CNRS UMR 8213, 10 rue Vauquelin 75005 Paris, France. \\ ${ }^{3}$ Sorbonne Université, CNRS, Institut Parisien de Chimie Moléculaire, IPCM, F-75005 Paris, \\ France. \\ ${ }^{4}$ Synchrotron-SOLEIL, Saint-Aubin, BP48, F91192 Gif sur Yvette Cedex, France.
}

* To whom correspondence should be sent: el@insp.upmc.fr

\begin{abstract}
Intraband and plasmonic transitions have appeared over the last years as an interesting tool to achieve optical absorption in the mid infrared. Tuning the doping magnitude has become a major challenge not only to tune the optical spectrum but also properties such as the dark current or the time response. Here we investigate the case of self-doped HgSe colloidal quantum dots (CQDs). Tuning of the doping was so far relying on band bending induced by a dipole design at the nanoparticle surface. With such a surface gating approach, it is difficult to conciliate both the massive tuning of the Fermi level with the preservation of transport properties of the CQD arrays. Here we propose a strategy to graft functionalized polyoxometalates (POMs) at the CQD surface and obtain simultaneously a massive tuning of the carrier density ( $\approx 5$ electrons per nanoparticle) and conduction properties. We bring a consistent demonstration of the HgSe CQD doping decrease by a charge transfer to the POM. This method is highly promising for large tuning of carrier density in degenerately doped semiconductor nanoparticles.
\end{abstract}




\section{INTRODUCTION}

Beyond their light emitting properties in the visible range, the broad tunability of optical absorption of colloidal quantum dots (CQDs) from UV to $\mathrm{THz}^{1}$ make them promising for the design of low-cost optoelectronic devices. Among potential applications of CQDs, solar cells ${ }^{2}$ and thermal imaging are two examples where infrared absorption is required. ${ }^{3}$ To push absorption of CQDs in the mid-infrared, two paths have been explored: either the use of interband transitions in narrow band gap semiconductors and semimetals or the use of doped semiconductors. ${ }^{4,5}$ In the latter case, intraband transition appears in the mid-infrared when the material becomes degenerately doped. Mercury chalcogenides selfdoped nanocrystals especially $\mathrm{HgS}^{6-8}{ }^{6 g S e} e^{9,10}$ and $\mathrm{HgTe}^{11}$ are certainly the most investigated materials because of their ability to combine intraband absorption in the mid-infrared with photoconductive properties. In the following we will focus on HgSe CQDs as model system for self-doped nanocrystals.

Self-doping ${ }^{4,12}$ of nanocrystals is a mechanism leading to degenerate doping ${ }^{13}$ which is specific to narrow band gap materials. Commonly doping is obtained through the introduction of extrinsic impurities into a semiconductor matrix (see the work on doped oxide ${ }^{14,15}$ ) or through the synthesis of non-stoichiometric material (this is the case of $\mathrm{Cu}_{2} \mathrm{~S}(\mathrm{e})^{16-18}$ ). In the case of mercury chalcogenides, the doping process is different. Indeed, most II-VI nanocrystals present a slight cation excess which leads to a n-type character. In the specific case of narrow band gap materials such as mercury chalcogenides, the conduction band state can be brought below the Fermi level of the environment: ${ }^{19}$ as a result the stable form of these CQDs is negatively charged and the 1Se, 1Pe and even 1De states can be filled with electrons, which corresponds to strongly degenerate doping.

The control of the doping magnitude becomes of utmost importance in photodetection applications, ${ }^{20}$ not only because it drives the optical spectrum but also because many other properties such as the dark current, the time response, their paramagnetic response...are connected to the exact number of carriers per particle. ${ }^{5,20}$ In particular infrared absorption based on intraband transition (moderate degenerate doping) and on plasmonic absorption (strongly degenerate doping) offer an interesting strategy to interband transition in narrow band gap material to address the mid infrared range of wavelengths. Because doping drives the optical spectrum in such materials, identifying a post synthesis method to tune it is of utmost interest.

Different strategies have been reported to tune the doping magnitude based on ligand exchange, ${ }^{19}$ heterostructure growth ${ }^{7,21}$ or nanoparticle size tuning. ${ }^{22,23}$ Among them, the surface gating consists in tuning the surface dipole at the nanoparticle surface to induce a band bending and thus control the relative doping. This approach has been widely used for PbS nanocrystals to change the nature of the majority carrier. ${ }^{24,25}$ In this direction, Kroupa et al have proposed to design molecules with especially large dipole. ${ }^{26}$ They reported a band displacement close to $2 \mathrm{eV}$. On the other hand in the case of mercury chalcogenides the variety of ligands which has been explored is much weaker and they typically consist in thiol-based molecules. ${ }^{23,27}$ In the case of HgSe nanoparticles, surface chemistry tuning has only led to a band shift of the order of $0.3 \mathrm{eV}$, which is nevertheless already close to the intraband transition energy. In this paper, we propose an alternative post synthesis method to boost the tunability of HgSe CQD doping, based on their hybridization with polyoxometalates (POMs).

POMs are molecular oxides formed by the early transition metals in their highest oxidation state $\left(\mathrm{W}^{\mathrm{V}}\right.$, $\mathrm{Mo}^{\mathrm{V}_{1}}$...). They are well known for being electron attractors ${ }^{28,29}$ and thus we can anticipate an electron transfer from the self-doped CQDs to the POMs. In this direction, a key challenge relies in the coupling between the POMs and the CQDs. There are a limited number of reports relative to this question ${ }^{30}$ and they are mostly motivated by the idea to use POMs as a charge transfer agent in dye sensitized solar cell. ${ }^{31,32}$ Here we take benefit of the great affinity of mercury for thiols and design a thiol functionalized 
POM. By using infrared spectroscopy, photoemission and transport measurement, we bring evidence for the electron transfer from the HgSe CQDs to the POMs and show for the first time a change of doping from 5 electrons per nanocrystal to less than 1 while tuning the surface chemistry.

\section{METHODS}

\section{Chemicals}

Mercury acetate $\left(\mathrm{Hg}(\mathrm{OAc})_{2}\right.$, Sigma-Amdrich), selenium powder (Se, Strem Chemicals), oleic acid (OA, Sigma-Aldrich, 90\%), oleylamine (OLA, Sigma-Aldrich, 80-90\%), trioctylphosphine (TOP, Sigma-Aldrich, 97\%), n-hexane (VWR, 99\%), ethanol absolute anhydrous (EtOH, Carlo Erba, 99.5\%), methanol (MeOH, Carlo Erba, 99.8\%), N,N-dimethylformamide (DMF, Sigma Aldrich), triethyloxonium tetrafluoroborate $\left(\mathrm{Et}_{3} \mathrm{O}^{+} \mathrm{BF}_{4}^{-}\right.$, Sigma Aldrich), lithium perchlorate $\left(\mathrm{LiClO}_{4}\right.$, Sigma-Aldrich, 98\%), 1,2-ethanedithiol (EDT, Fluka, 98\%), polyethylene glycol (PEG 6k, $\mathrm{M}_{\mathrm{w}}=6.10^{3} \mathrm{~g} \cdot \mathrm{mol}^{-1}$, Fluka).

\section{Material synthesis}

HgSe QD synthesis: In a $50 \mathrm{~mL}$ three neck flask, $0.5 \mathrm{~g}$ of mercury acetate $\left(\mathrm{Hg}(\mathrm{OAc})_{2}\right), 10 \mathrm{~mL}$ of oleic acid (OA) and $25 \mathrm{~mL}$ of oleylamine (OLA) are degassed at $85^{\circ} \mathrm{C}$ for $1 \mathrm{~h}$. Under argon atmosphere at 90 ${ }^{\circ} \mathrm{C}, 1.6 \mathrm{~mL}$ of a $1 \mathrm{M}$ solution of Selenium (Se) in trioctylphosphine (TOP) is quickly injected into the flask. After $4 \mathrm{~min}$, the reaction is quenched by adding quickly $10 \mathrm{~mL}$ of hexane and cooled down to room temperature using ice bath. The obtained dark solution is then opened to air and precipitated by addition of ethanol and methanol and then centrifuged. The supernatant is discarded and the pellet is redispersed in hexane. The cleaning procedure is repeated two more times. At the last step, we perform a selective precipitation to split the batch over two fractions. To do so, the solution in pure hexane is first centrifuged without the addition of non-solvent to get rid of the lamellar phase resulting from the complexation of mercury with OLA. Then, only the stable solution of HgSe in hexane is kept while the gray precipitate is discarded. The nanoparticles in this fraction are $5 \pm 0.7 \mathrm{~nm}$, measured by transmission electron microscopy (TEM), see Figure $1 \mathrm{~b}$. The intraband absorption peak is at $2080 \mathrm{~cm}^{-}$ 1 , see Figure 1c. Because of the absence of DDT ligands, the obtained solution is poorly stable, and the HgSe CQDs can no longer be redispersed in hexane after 30 minutes.

POM-SH (TBA $)_{3}\left[\mathrm{PW}_{11} \mathrm{O}_{39}\left\{\mathrm{O}\left(\mathrm{SiC}_{3} \mathrm{H}_{6} \mathrm{SH}\right)_{2}\right\}\right]$ synthesis: $\mathrm{K}_{7}\left[\mathrm{PW}_{11} \mathrm{O}_{39}\right](0.64 \mathrm{~g}, 0.2 \mathrm{mmol})$ is dissolved in a water/acetonitrile mixture $(30 \mathrm{~mL}, 1: 1)$. A $1 \mathrm{M} \mathrm{HCl}$ aqueous solution is added drop by drop until an apparent $\mathrm{pH}$ equals to 3 . The solution is cooled in an ice bath and the $\left(\mathrm{OCH}_{3}\right)_{3} \mathrm{SiC}_{3} \mathrm{H}_{6} \mathrm{SH}(0.157 \mathrm{~mL}, 0.8$ $\mathrm{mmol}$ ) is inserted. The $1 \mathrm{M} \mathrm{HCl}$ solution is added drop by drop again to reach $\mathrm{pH}_{\mathrm{app}}=2$. After an all night reaction, $\operatorname{TBABr}(0.26 \mathrm{~g}, 0.8 \mathrm{mmol})$ is added and the solution concentrated with a rotary evaporator to precipitate the product. The sticky solid is dissolved in the minimum of acetonitrile then precipitated again with an excess of diethylether. The solid is recovered by centrifugation and washed thoroughly with diethylether to obtain a white powder $(0.55 \mathrm{~g}, 76 \%)$.

${ }^{1} \mathrm{H}$ NMR $\left(400 \mathrm{MHz}, \mathrm{CD}_{3} \mathrm{CN}\right): \delta(\mathrm{ppm}) 3.12(\mathrm{~m}, 24 \mathrm{H}), 2.64(\mathrm{~m}, 4 \mathrm{H}), 1.87(\mathrm{~m}, 4 \mathrm{H}), 1.63(\mathrm{~m}, 25 \mathrm{H}), 1.40$ (sex, $3 \mathrm{~J}(\mathrm{H}, \mathrm{H})=7.4 \mathrm{~Hz}, 24 \mathrm{H}), 0.99(\mathrm{t}, 3 \mathrm{~J}(\mathrm{H}, \mathrm{H})=7.4 \mathrm{~Hz}, 36 \mathrm{H}), 0.90(\mathrm{~m}, 4 \mathrm{H})$;

${ }^{31}$ P NMR (121 MHz, CD3CN) $\delta$ (ppm) -12.37; IR (KBr pellet) : $\delta=2962(\mathrm{~m}), 2934(\mathrm{~m}), 2873(\mathrm{w}), 1483(\mathrm{~m})$, $1381(\mathrm{w}), 1111$ (s), 1065 (s), 1052 (s), 1036 (s), 963 (vs), 870 (vs), 824 (vs), 711 (m), 585 (w), 522 (m), $383(\mathrm{~s})$;

MS (ESI-), m/z (\%): calculated for $\mathrm{C}_{6} \mathrm{H}_{14} \mathrm{O}_{40} \mathrm{PS}_{2} \mathrm{Si}_{2} \mathrm{~W}_{11}: 966.56[\mathrm{M}]^{4-}$; found : 966.75 (100) 
Elemental Analysis: calculated (\%) for $\mathrm{C}_{70} \mathrm{H}_{158} \mathrm{~N}_{4} \mathrm{O}_{40} \mathrm{~S}_{2} \mathrm{Si}_{3} \mathrm{~W}_{11}$ : C 21.74, H 4.09, N 1.45; found: $\mathrm{C} 21.70, \mathrm{H}$ $3.97, \mathrm{~N} 1.43$

$(\mathrm{TBA})_{3}\left[\mathrm{PW}_{12} \mathrm{O}_{40}\right]^{33}$ and $\mathrm{K}_{7}\left[\mathrm{PW}_{11} \mathrm{O}_{39}\right]^{34}$

was synthesized according to published procedures.

OA/OLA towards POM-SH ligand exchange procedure: $500 \mu \mathrm{L}$ of the solution of fresh HgSe QDs in hexane $\left(10 \mathrm{mg} \cdot \mathrm{mL}^{-1}\right)$ is mixed with $1 \mathrm{~mL}$ of a solution of triethyloxonium tetrafluoroborate in DMF (50 $\mathrm{mg} \cdot \mathrm{mL}^{-1}$ ) to clean $\mathrm{QD}$ surface of any ligand. After vigorous agitation and sonication, the solution is centrifuged for $2 \mathrm{~min}$ at $5500 \mathrm{rpm}$. The supernatant is discarded while the QDs in the pellet are redispersed in $0.5 \mathrm{~mL}$ of a solution of POM-SH in DMF $\left(50 \mathrm{mg} \cdot \mathrm{mL}^{-1}\right)$. The obtained solution is more colloidably stable than the initial HgSe QDs in hexane without the POM-SH. To remove the excess of POM-SH, the solution is centrifuged at $5500 \mathrm{rpm}$ for $10 \mathrm{~min}$. The POM-SH capped QDs fall in the pellet while the excess of POM-SH stay in the supernatant. The supernatant is then discarded and the pellet is redispersed in fresh DMF. The obtained solution is colloidably stable (several months).

OA/OLA towards EDT ligand exchange procedure: Solution of HgSe QDs in hexane is dropcasted on a clean substrate. Once the solvent is completely evaporated, ligand exchange is performed on the sample to remove OA/OLA and replace it by EDT. The EDT ligands are simply dissolved in ethanol to reach a $1 \mathrm{wt} \%$ solution. To perform ligand exchange, the film is dipped into the EDT solution for $90 \mathrm{~s}$. Then the film is rinsed with ethanol for $30 \mathrm{~s}$.

\section{Transport measurement}

Electrode fabrication: Electrodes are fabricated using standard optical lithography methods. Briefly, the surface of a Si/SiO $/ 400 \mathrm{~nm}$ thick) wafer is cleaned by sonication in acetone. The wafer is rinsed with isopropanol and finally cleaned using an $\mathrm{O}_{2}$ plasma. AZ5214 resist is spin-coated and baked at 110 ${ }^{\circ} \mathrm{C}$ for $90 \mathrm{~s}$. The substrate is exposed under UV through a pattern mask for $1.5 \mathrm{~s}$. The film is further baked at $125{ }^{\circ} \mathrm{C}$ for $2 \mathrm{~min}$ to invert the resist. Then, a $40 \mathrm{~s}$ flood exposure is performed. The resist is developed using a bath of AZ726 for $32 \mathrm{~s}$, before being rinsed with pure water. We then deposit a 5 $\mathrm{nm} \mathrm{Cr}$ layer and a $80 \mathrm{~nm}$ gold layer using a thermal evaporator. The lift-off is performed by dipping the film for $1 \mathrm{~h}$ in acetone. The electrodes are finally rinsed using isopropanol and dried by air flow. The electrodes are $2.5 \mathrm{~mm}$ long and spaced by $20 \mu \mathrm{m}$.

Electrolyte preparation: $\mathrm{LiClO}_{4}(500 \mathrm{mg})$ is mixed with $2.3 \mathrm{~g}$ of polyethylene glycol on a hot plate in an argon-filled glove box at $170^{\circ} \mathrm{C}$ for $2 \mathrm{~h}$.

Low temperature DC transport: Temperature-dependent electrical measurements were carried out inside a He-flow cryostat, using a low current SourceMeter Keithley 2634B.

Transistor measurements: Transistor measurements are conducted in Ar filled glove box using two Keithley 2400 as source-meters, by biasing the drain source under low bias (between 5 and $100 \mathrm{mV}$ ) and applying a varying gate bias. The gate step bias is $1 \mathrm{mV}$ at $2 \mathrm{mV} . \mathrm{s}^{-1}$ for electrolyte gating. A scheme of the setup is presented on Figure $3 a$.

\section{DISCUSSION}

We first synthetize $5 \mathrm{~nm}$ HgSe CQDs presenting intraband absorption feature at $\approx 2000 \mathrm{~cm}^{-1}$ using dodecanethiol ligands at the end of the reaction to ensure HgSe CQDs a strong colloidal stability. ${ }^{9}$ The 
infrared spectrum of this material presents two main features. In the near infrared (wavenumber above $6000 \mathrm{~cm}^{-1}$ ), there is an absorption edge corresponding to interband transition. In the mid infrared (from 3000 to $2000 \mathrm{~cm}^{-1}$ ), there is a narrow band absorption which corresponds to intraband features. The spectrum of this material is presented in reference ${ }^{23}$ and is very similar to the one presented on Figure 1c (with different capping ligands). According to previous combined photoemission and infrared spectroscopy study, these HgSe CQDs are electron rich with typically the $1 \mathrm{~S}_{\mathrm{e}}$ fully filled and the $1 \mathrm{P}_{\mathrm{e}}$ partly filled state once capped with their native dodecanthiol ligand ${ }^{23} .1 \mathrm{~S}_{\mathrm{e}}$ and the $1 \mathrm{P}_{\mathrm{e}}$ are respectively twice and six times degenerated, thus a full filling of these two states corresponds to 8 electrons per nanocrystal. ${ }^{23}$ Assuming a randomly close packing for the CQDs, this correspond to carrier density of $\approx 8 \times 10^{19}$ carrier per $\mathrm{cm}^{-3}$.

We then synthetize POMs with tungsten as metal, stabilized with tetrabutylammonium (TBA) (see Supporting Information for more information about synthesis and structure). The POMs have the following stoichiometry (TBA) ${ }_{3} \mathrm{PW}_{12} \mathrm{O}_{40}$, where $\mathrm{W}$ atoms are highly oxidized (+VI oxidation state) and so can be used as electron attractors when grafted onto HgSe QDs.

In order to couple the POMs onto the HgSe CQD surface, we first try the method proposed by Huang et $a .^{30}$ This procedure consists in two steps: first, the native ligands are removed from CQD surface using an alkylating agent; then the POMs are grafted onto QD nude surface. In our case, this procedure has been unsuccessful: we observe that the final solution of POM capped HgSe QDs is not stable in DMF despite the high solubility of POMs in this solvent, meaning that the grafting of ligands is not done. Moreover we notice that the alkylating agent that we use (triethyloxonium tetrafluoroborate, $\mathrm{EtO}_{3}{ }^{+} \mathrm{BF}_{4}{ }^{-}$) cannot remove the native dodecanethiol ligands at the surface of $\mathrm{HgSe}$ nanocrystals, probably due to the great affinity of mercury with sulfur.

Consequently, we update the HgSe CQD synthesis to get rid of any thiol ligand and replace them by oleylamine and oleic acid. The obtained nanocrystals keep a spherical shape despite the change of ligands (see Figure 1b), but their colloidal stability is very poor: typically after 30 minutes, the CQDs aggregate and can no longer be redispersed even with sonication. In addition, we choose to increase the affinity of the HgSe CQDs for the POM, by using a silyl-substituted POM terminated by two thiol groups $(\mathrm{TBA})_{3}\left[\mathrm{PW}_{11} \mathrm{O}_{40}\left(\mathrm{SiC}_{3} \mathrm{H}_{6} \mathrm{SH}\right)_{2}\right](\mathrm{POM}-\mathrm{SH})$. Using these POM-SH ligands, we successfully attach them at the surface of HgSe CQDs (a scheme of the obtained QDs is presented on Figure 1a) using a phase transfer ${ }^{35}$ procedure and obtain at the end a stable colloidal solution in DMF. Note that we estimate the number of grated POM per QD to be in the 1 to 10 range. 


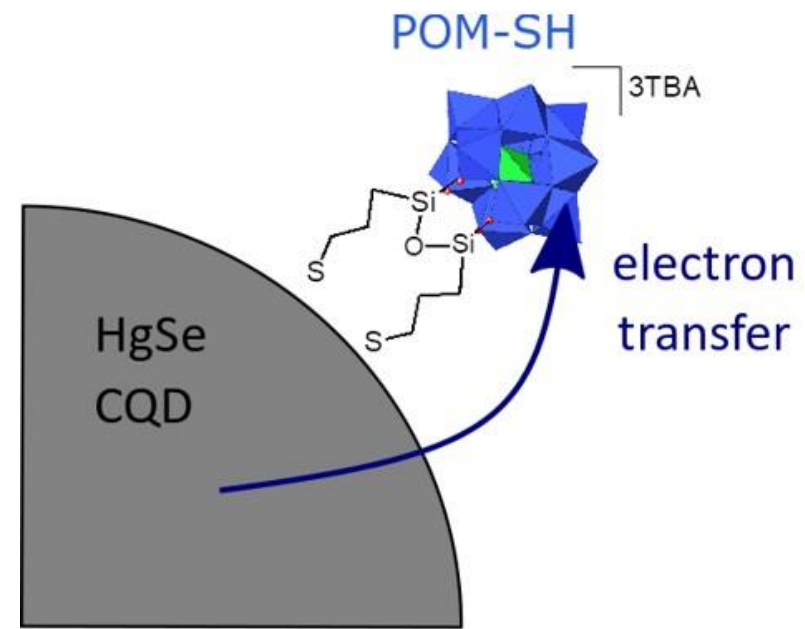

a.

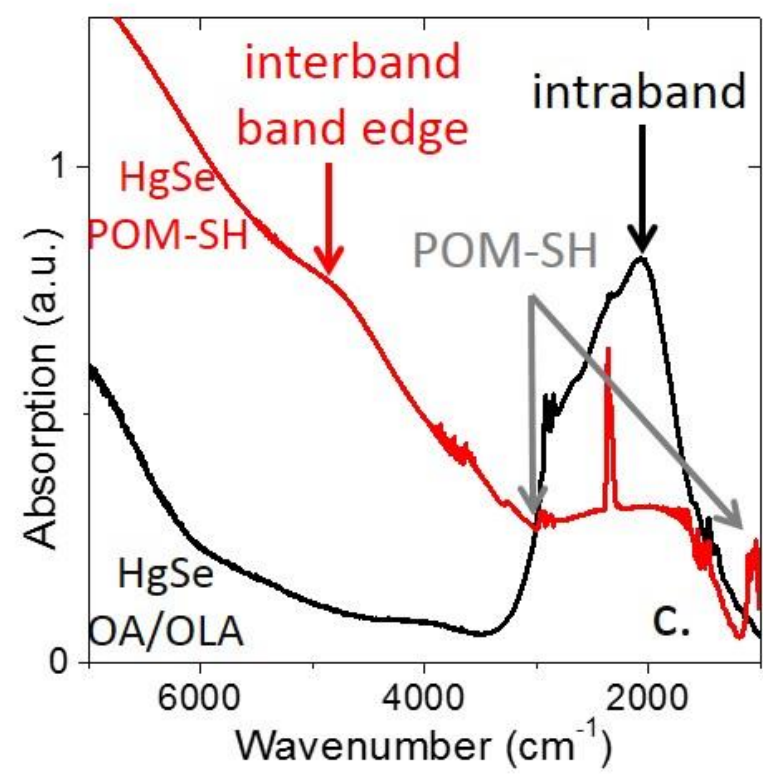

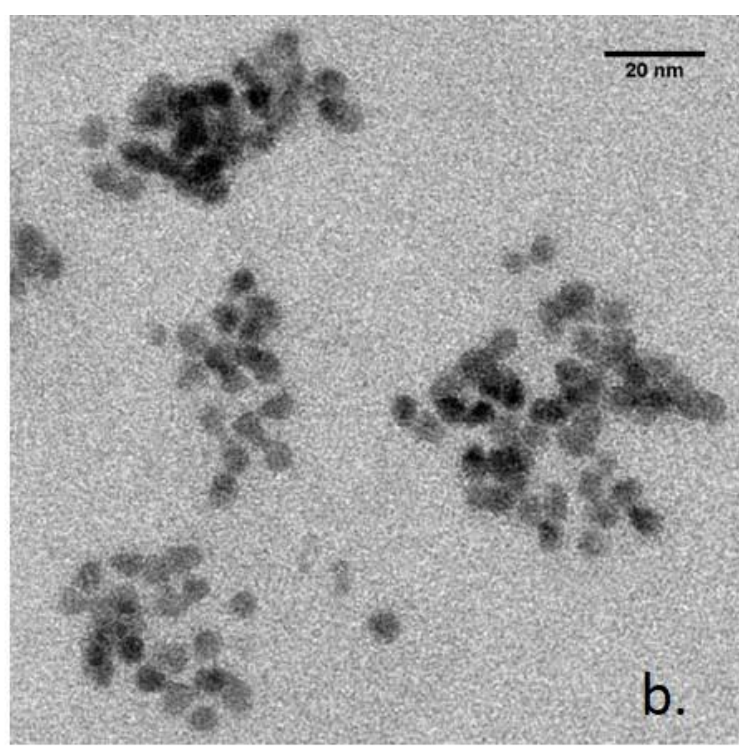

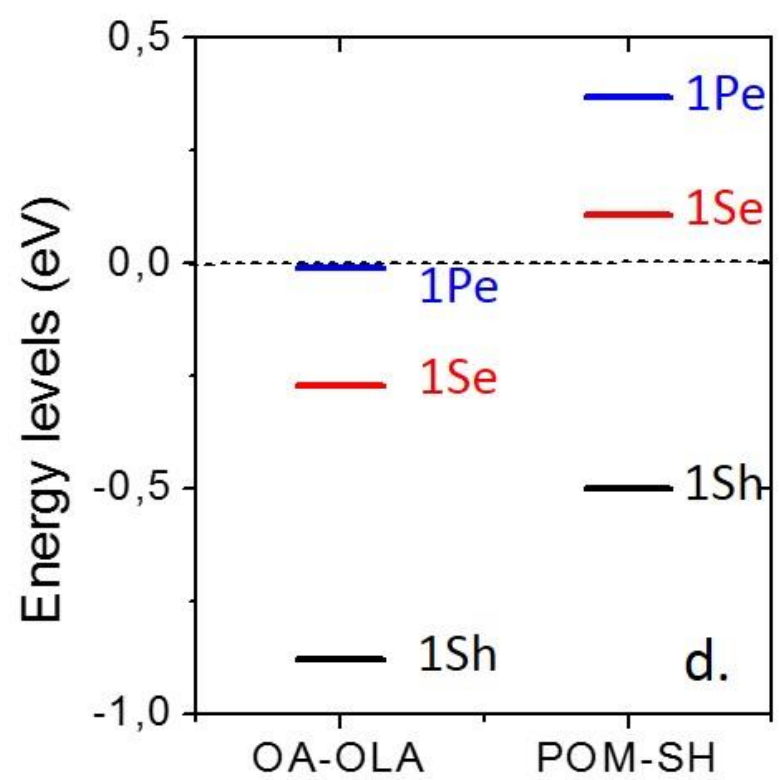

Figure 1: a. Scheme of HgSe QDs capped with POM-SH ligands. The arrow corresponds to the electron transfer from doped HgSe QD to electron attractor POM-SH ligand. b. TEM picture of HgSe QDs capped with POM-SH. c. FTIR spectra of HgSe QDs just after synthesis (in black) and after POM-SH grafting at the surface (in red). OA and OLA refer respectively to oleic acid and oleylamine, the only ligands used in the synthesis. Note that the peak at $\approx 2300 \mathrm{~cm}^{-1}$ is due to $\mathrm{CO}_{2}$. d. Energy levels of HgSe after synthesis (OA-OLA) and after POM-SH grafting. The dot line corresponds to Fermi level which is used as reference at 0 $\mathrm{eV} .1 \mathrm{Se}$ and $1 \mathrm{Pe}$ correspond respectively to first and second level of conduction band; $1 S_{h}$ corresponds to last level of valence band.

As POMs are electron attractors, ${ }^{28,29}$ we investigated their influence on the infrared spectrum of the HgSe CQDs and more specifically on their intraband feature. To do so, we recorded transmission spectra before and after POM-SH grafting, see Figure 1c. With their oleic acid (OA) and oleylamine (OLA) capping ligands, the HgSe CQDs present interband transition at wavenumbers above $6000 \mathrm{~cm}^{-1}$ which does not correspond to the band-edge feature ${ }^{23}$ as it is fully bleached (ie the $1 \mathrm{~S}_{\mathrm{e}}$ level is full) but to the transition between valence band and deeper levels in the conduction band. Around $2000 \mathrm{~cm}^{-1}$, the intraband peak is clearly noticeable. After POM grafting, the intraband feature at $2000 \mathrm{~cm}^{-1}$ mostly disappears whereas the interband band-edge feature emerges at $4900 \mathrm{~cm}^{-1}$, meaning that the transition between $1 \mathrm{~S}_{\mathrm{h}}$ and $1 \mathrm{~S}_{\mathrm{e}}$ level is authorized. Additional peak at $3000 \mathrm{~cm}^{-1}$ and below $1500 \mathrm{~cm}^{-1}$ also clearly appear and can be attributed respectively to the organic tether, the counter cations and to 
the POM itself (see figure S1). This change of absorption weight between the band edge feature and the intraband feature is a clear signature of the dedoping of the CQDs ${ }^{36}$ : the removing of electrons from conduction band levels leads to disappearance of intraband feature and unbleaching of the band edge absorption. It is also worth noting that evidence for the charge transfer from the QD to the POM is still preserved while the material is under film form. This is a key difference with previous reports on molecule to QD charge transfer which was possible in solution but unobserved while the QD where under a solid array form. ${ }^{37}$ The fact that charge transfer gets preserved under solid form is quite promising for the use of such doping control method at the device scale

To further clarify the impact of the POMs on the carrier density of the HgSe CQDs, we conduct photoemission measurements on the TEMPO beamline of Synchrotron Soleil. The low binding energy part of the photoemission spectrum is used to reveal the position of the valence band edge with respect to the Fermi level. We obtain a value of $0.88 \mathrm{eV}$ for the HgSe CQDs capped with their native dodecanethiol ligand (see Figure S2). This value has to be compared to the interband edge energy $\approx 0.60 \mathrm{eV}$ confirming that initial HgSe CQDs are degenerately doped with the Fermi level deeply in the conduction band. This is consistent with the observation of the intraband feature on the infrared spectrum. After POM grafting, the valence band is only $0.5 \mathrm{eV}$ below the Fermi level, meaning that the Fermi level is now within the band gap. Again this observation is consistent with the disappearance of the intraband feature and the recovery of the band edge transition. To further illustrate this shift of the band with respect to the Fermi level, the reconstructed energy levels in absolute energy for both materials are presented on Figure $1 \mathrm{~d}$. This graph demonstrates the possibility to tune the doping of $\mathrm{HgSe}$. Initially the Fermi level is resonant with the $1 \mathrm{Pe}$ state, meaning that the latter is half filled. Since this state is 6 fold degenerated, there are 3 electrons in the $1 \mathrm{P}_{\mathrm{e}}$ state and 2 more in the $1 \mathrm{~S}_{\mathrm{e}}$ state for a total of 5 electrons per nanoparticle. After the grafting of the POM, the Fermi level is brought within the band gap and we can estimate the thermal population of the 1Se state to be given by 2. $\exp \left(-\frac{E_{1 S e}-E f}{k b T}\right)=0.03$ electron/nanoparticle with $E_{1 S e}-E f=107 \mathrm{meV}$ the difference in energy between the $1 \mathrm{~S}_{\mathrm{e}}$ state and the Fermi level and $\mathrm{kbT}$ the thermal energy ( $\approx 25 \mathrm{meV}$ at room temperature). Almost 5 electrons per nanoparticle are transferred toward the POM, which is by far the greatest tuning of the carrier density reported for self-doped CQDs.

In order to confirm that HgSe CQD change of doping is the result of the POM grafting, we investigate the photoemission signal of the pristine HgSe CQDs, of the pristine POM-SH and of the grafted POM on HgSe CQDs. Experimental data are given in Figure 2.

The overviews obtained for both films are presented on Figure 2a. For POM-SH capped HgSe QDs, the presence of tungsten coming from POM-SH ligands on the overview confirms that the POM-SH are present into the sample. The observation of the $\mathrm{W} 4 \mathrm{f}$ core level from the pristine POM-SH ligands reveals the presence of a single contribution at a binding energy of $36.03 \mathrm{eV}$ (see Figure $2 \mathrm{~b}$ ). This value is consistent with the $+\mathrm{VI}$ oxidation state of $\mathrm{W} .{ }^{38}$ The charge transfer from the HgSe electron rich CQDs to the oxidized POM is well evidenced by investigating the spectrum relative to the $\mathrm{W} 4 \mathrm{f}$ of the hybrid material, see Figure $2 \mathrm{~b}$. This state can no longer be fitted by a single contribution. More specifically, new contributions appear at lower binding energy, which means that $W$ presents contribution from lower oxidation states ${ }^{39,40}$ typically $+\mathrm{V}$ and possibly $+\mathrm{IV}$. This multielectronic reduction of the POM-SH is a clear signature of a charge transfer ant that the POM-SH is responsible for the de-doping of the HgSe CQDs. 

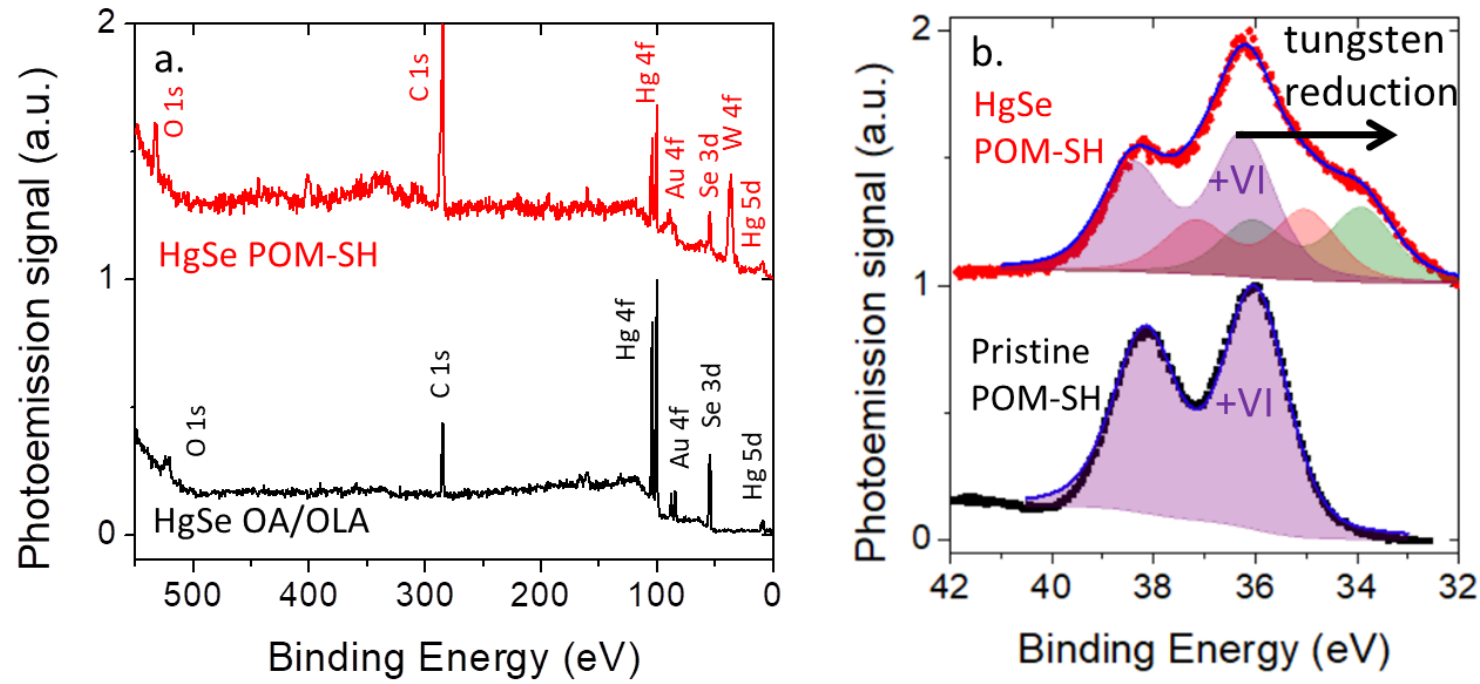

Figure 2: XPS overviews of HgSe capped with OA/OLA (in black) and of HgSe capped with POM-SH (in red). The samples are deposited on gold substrate, which leads to the Au4f signal around $84 \mathrm{eV} . \mathrm{b}$. W $4 \mathrm{f}$ core level of the POM-SH. Three doublets are necessary to fit this core level in the case of POM-SH capped HgSe, which are at lower binding energies so corresponding to lower oxidation states.

In the following, we investigate how this modification of the HgSe CQD carrier density is impacting the transport properties of HgSe CQD arrays. HgSe is typically expected to be used as active material for low cost photodetection. ${ }^{5,9} \mathrm{~A}$ key figure of merit is dark current. While doping is important to obtain intraband transition, it is also driving the magnitude of the dark current, reducing doping is then of utmost interest. In the case of OA/OLA capping ligands, we exchange OA/OLA with ethanedithiol ligands using the procedure described in the Supporting Information. This ligand exchange has very little effect on the doping level, as shown by Martinez et al. ${ }^{23}$ On the other hand, in the case of POM capped HgSe CQDs, we just deposited the QDs + DMF solution and dried at $100{ }^{\circ} \mathrm{C}$ for a minute to evaporate the solvent.

It was a priori unclear if the POM grafted HgSe CQD films were conductive or not. Indeed the large size of the POM may have prevented the coupling of the CQDs, leading to a poor hopping transport efficiency. Nevertheless, a conductive film is obtained.

Transport is probed in an electrolyte gating field effect transistor configuration, ${ }^{41,42}$ see Figure $3 a$ for a scheme of the device. The main motivation for such a gating approach comes from the ability of the electrolyte to inject a large number of carriers per nanoparticle. This is of utmost importance to tune the carrier density of strongly doped CQDs. Without POM, the HgSe CQDs show only a n-type behavior with a negative threshold voltage (ie the material is inherently doped), characterized by a rise of conductance while increasing gate bias, see Figure $3 \mathrm{~b}$. No hole transport is observed, which is consistent with the electron degenerate doping of this material.

While POM-SH are grafted on the CQD surface, the transfer curve (drain current as a function of gate bias) is strongly affected. The material turns to be ambipolar (see Figure $3 c$ ) with both hole and electron conductions. This confirms that the Fermi level is now closer to the valence band than it is without POMs.

The reduction of doping is also affecting the temperature dependence of the material. HgSe with conventional ligands typically suffer from very weak temperature dependence. Typically the Arrhenius fit of the current as a function of temperature in the vicinity of the room temperature leads to an activation energy ( $18 \mathrm{meV}$ ) even smaller than $k_{b} T$ with $k_{b}$ being the Boltzmann constant, see Figure $3 \mathrm{~d}$. 
As a result, cooling the sample is not a viable strategy to reduce the dark current and improve photodetection performances. In the presence of POM-SH ligands, the activation energy $(50 \mathrm{meV})$ is increased by a factor 2.5, Figure $3 \mathrm{~d}$. This is again consistent with the displacement of the Fermi level in a part of the electronic spectrum where the density of states is sparser.
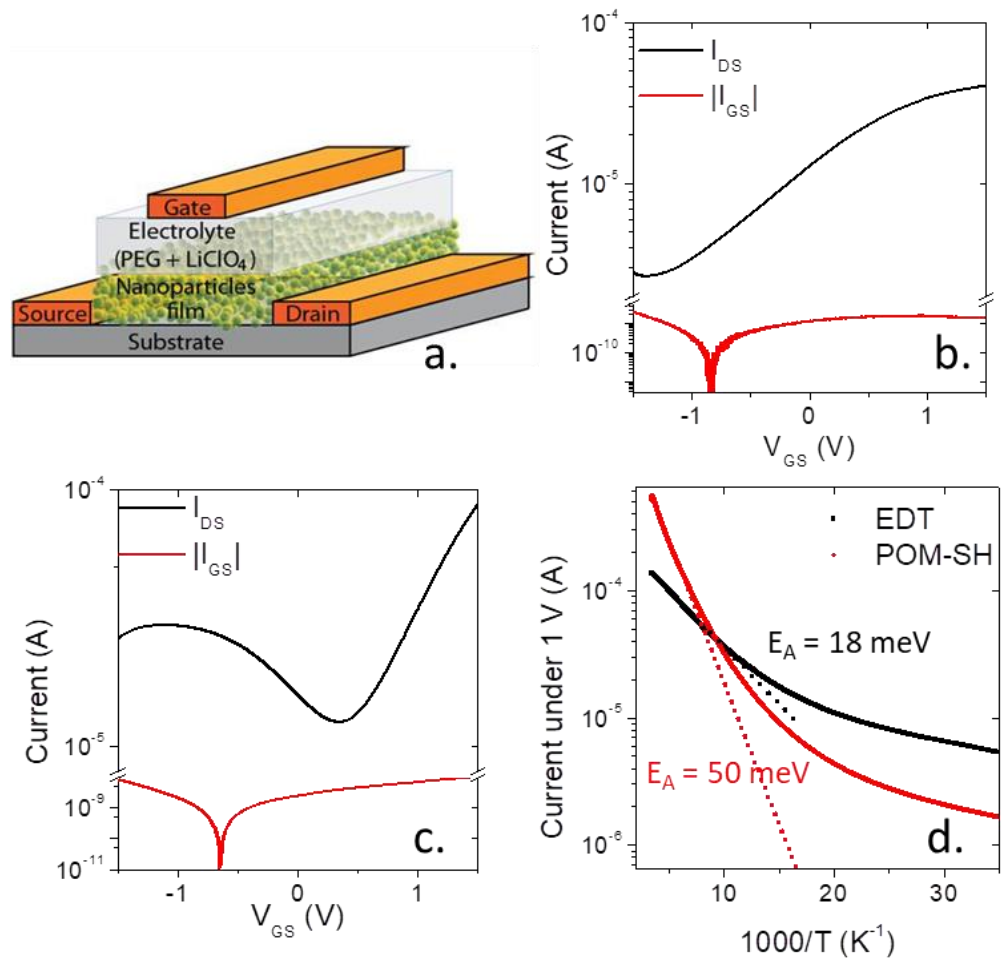

Figure 3: Transport measurements on HgSe CQD thin films. a. Scheme of the field-effect transistor set-up. ${ }^{41}$ In the following, the source is named S, the drain D and the gate G. b. FET measurement made on HgSe capped with EDT, $V_{D S}=5 \mathrm{mV}$. c. FET measurement made on HgSe capped with POM-SH, $V_{D S}=100 \mathrm{mV}$. d. Current as a function of decreasing temperature, $V_{D S}=1$ $V$. The solid curves correspond to raw data and the dot curves correspond to Arrhenius fit.

\section{ASSOCIATED CONTENT}

Supporting Information

The Supporting Information is available free of charge on the ACS Publications website at DOI:

POM SH characterization, details for material characterization methods, and photoemission measurements.

\section{CONCLUSION}

Control of doping is of utmost importance for the use of doped CQDs in application where intraband and plasmonic transition appear. Previously the main strategy for tuning the doping was relying on surface gating based on the design of dipole ${ }^{19,25,26}$ to induce band bending at the nanocrystal surface. Here we demonstrate that the grafting of electron attractor molecules such as POMs is a viable way for much broader tuning of the carrier density (here up to 5 carriers per nanoparticle). All infrared spectroscopy, photoemission and transport measurements bring a consistent picture for a charge transfer from the CQDs to the POMs leading to (i) doping decrease in the CQDs, (ii) reduction of the POM. Moreover we demonstrate that the presence of the POMs is compatible with electronic transport in POM-CQD hybrid array. This strategy is not limited to mercury chalcogenides and is also 
promising for the tuning of doping in degenerately doped nanoparticles such as oxide or silicon nanoparticles.

\section{ACKNOWLEDEGMENTS}

EL thanks the support ERC starting grant blackQD (grant $n^{\circ} 756225$ ). We acknowledge the use of cleanroom facilities from the "Centrale de Proximite Paris-Centre". This work has been supported by the Region Ile-de-France in the framework of DIM Nano-K (grant dopQD). This work was supported by French state funds managed by the ANR within the Investissements d'Avenir programme under reference ANR-11-IDEX-0004-02, and more specifically within the framework of the Cluster of Excellence MATISSE and also by the grant Nanodose and H2DH.

\section{REFERENCES}

(1) Goubet, N.; Jagtap, A.; Livache, C.; Martinez, B.; Portalès, H.; Xu, X. Z.; Lobo, R. P. S. M.; Dubertret, B.; Lhuillier, E. Terahertz HgTe Nanocrystals: Beyond Confinement. J. Am. Chem. Soc. 2018, 140 (15), 5033-5036.

(2) McDonald, S. A.; Konstantatos, G.; Zhang, S.; Cyr, P. W.; Klem, E. J. D.; Levina, L.; Sargent, E. H. Solution-Processed PbS Quantum Dot Infrared Photodetectors and Photovoltaics. Nat. Mater. 2005, 4, 138-142.

(3) Lhuillier, E.; Guyot-Sionnest, P. Recent Progresses in Mid Infrared Nanocrystal Based Optoelectronics. IEEE J. Sel. Top. Quantum Electron. 2017, PP (99), 1-1.

(4) Jagtap, A.; Livache, C.; Martinez, B.; Qu, J.; Chu, A.; Gréboval, C.; Goubet, N.; Lhuillier, E. Emergence of Intraband Transitions in Colloidal Nanocrystals. Opt. Mater. Express 2018, 8 (5), 1174-1183.

(5) Deng, Zhiyou; Jeong, Kwang Seob; Guyot-Sionnest, Philippe. Colloidal Quantum Dots Intraband Photodetectors. ACS Nano 2014, 8 (11), 11707-11714.

(6) Kim, J.; Yoon, B.; Kim, J.; Choi, Y.; Kwon, Y.-W.; Kyu Park, S.; Seob Jeong, K. High Electron Mobility of $\beta$-HgS Colloidal Quantum Dots with Doubly Occupied Quantum States. RSC Adv. 2017, 7 (61), 38166-38170.

(7) Shen, G.; Guyot-Sionnest, P. HgS and HgS/CdS Colloidal Quantum Dots with Infrared Intraband Transitions and Emergence of a Surface Plasmon. J. Phys. Chem. C 2016, 120 (21), 11744-11753.

(8) Jeong, K. S.; Deng, Z.; Keuleyan, S.; Liu, H.; Guyot-Sionnest, P. Air-Stable N-Doped Colloidal HgS Quantum Dots. J. Phys. Chem. Lett. 2014, 5 (7), 1139-1143.

(9) Lhuillier, E.; Scarafagio, M.; Hease, P.; Nadal, B.; Aubin, H.; Xu, X. Z.; Lequeux, N.; Patriarche, G.; Ithurria, S.; Dubertret, B. Infrared Photodetection Based on Colloidal Quantum-Dot Films with High Mobility and Optical Absorption up to THz. Nano Lett. 2016, 16 (2), 1282-1286.

(10) Wang, H.; Lhuillier, E.; Yu, Q.; Zimmers, A.; Dubertret, B.; Ulysse, C.; Aubin, H. Transport in a Single Self-Doped Nanocrystal. ACS Nano 2017, 11 (2), 1222-1229.

(11) Shen, G.; Chen, M.; Guyot-Sionnest, P. Synthesis of Nonaggregating HgTe Colloidal Quantum Dots and the Emergence of Air-Stable N-Doping. J. Phys. Chem. Lett. 2017, 8 (10), 2224-2228.

(12) Kim, J.; Choi, D.; Jeong, K. S. Self-Doped Colloidal Semiconductor Nanocrystals with Intraband Transitions in Steady State. Chem. Commun. 2018, 54 (61), 8435-8445.

(13) Araujo, J. J.; Brozek, C. K.; Kroupa, D.; Gamelin, D. R. Degenerately N-Doped Colloidal PbSe Quantum Dots: Band Assignments and Electrostatic Effects. Nano Lett. 2018.

(14) Luther, J. M.; Jain, P. K.; Ewers, T.; Alivisatos, A. P. Localized Surface Plasmon Resonances Arising from Free Carriers in Doped Quantum Dots. Nat. Mater. 2011, 10 (5), 361-366.

(15) Agrawal, A.; Johns, R. W.; Milliron, D. J. Control of Localized Surface Plasmon Resonances in Metal Oxide Nanocrystals. Annu. Rev. Mater. Res. 2017, 47 (1), 1-31. 
(16) Kriegel, I.; Jiang, C.; Rodríguez-Fernández, J.; Schaller, R. D.; Talapin, D. V.; da Como, E.; Feldmann, J. Tuning the Excitonic and Plasmonic Properties of Copper Chalcogenide Nanocrystals. J. Am. Chem. Soc. 2012, 134 (3), 1583-1590.

(17) Coughlan, C.; Ibáñez, M.; Dobrozhan, O.; Singh, A.; Cabot, A.; Ryan, K. M. Compound Copper Chalcogenide Nanocrystals. Chem. Rev. 2017, 117 (9), 5865-6109.

(18) Dorfs, D.; Härtling, T.; Miszta, K.; Bigall, N. C.; Kim, M. R.; Genovese, A.; Falqui, A.; Povia, M.; Manna, L. Reversible Tunability of the Near-Infrared Valence Band Plasmon Resonance in Cu2xSe Nanocrystals. J. Am. Chem. Soc. 2011, 133 (29), 11175-11180.

(19) Robin, A.; Livache, C.; Ithurria, S.; Lacaze, E.; Dubertret, B.; Lhuillier, E. Surface Control of Doping in Self-Doped Nanocrystals. ACS Appl. Mater. Interfaces 2016, 8 (40), 27122-27128.

(20) Jeong, J.; Yoon, B.; Kwon, Y.-W.; Choi, D.; Jeong, K. S. Singly and Doubly Occupied Higher Quantum States in Nanocrystals. Nano Lett. 2017, 17 (2), 1187-1193.

(21) Sagar, L. K.; Walravens, W.; Maes, J.; Geiregat, P.; Hens, Z. HgSe/CdE (E = S, Se) Core/Shell Nanocrystals by Colloidal Atomic Layer Deposition. J. Phys. Chem. C 2017, 121 (25), 1381613822.

(22) Chen, M.; Guyot-Sionnest, P. Reversible Electrochemistry of Mercury Chalcogenide Colloidal Quantum Dot Films. ACS Nano 2017, 11 (4), 4165-4173.

(23) Martinez, B.; Livache, C.; Notemgnou Mouafo, L. D.; Goubet, N.; Keuleyan, S.; Cruguel, H.; Ithurria, S.; Aubin, H.; Ouerghi, A.; Doudin, B.; et al. HgSe Self-Doped Nanocrystals as a Platform to Investigate the Effects of Vanishing Confinement. ACS Appl. Mater. Interfaces 2017, 9 (41), 36173-36180.

(24) Miller, E. M.; Kroupa, D. M.; Zhang, J.; Schulz, P.; Marshall, A. R.; Kahn, A.; Lany, S.; Luther, J. M.; Beard, M. C.; Perkins, C. L.; et al. Revisiting the Valence and Conduction Band Size Dependence of PbS Quantum Dot Thin Films. ACS Nano 2016, 10 (3), 3302-3311.

(25) Brown, P. R.; Kim, D.; Lunt, R. R.; Zhao, N.; Bawendi, M. G.; Grossman, J. C.; Bulović, V. Energy Level Modification in Lead Sulfide Quantum Dot Thin Films through Ligand Exchange. ACS Nano 2014, $8(6)$, 5863-5872.

(26) Kroupa, D. M.; Vörös, M.; Brawand, N. P.; McNichols, B. W.; Miller, E. M.; Gu, J.; Nozik, A. J.; Sellinger, A.; Galli, G.; Beard, M. C. Tuning Colloidal Quantum Dot Band Edge Positions through Solution-Phase Surface Chemistry Modification. Nat. Commun. 2017, 8, 15257.

(27) Martinez, B.; Livache, C.; Goubet, N.; Jagtap, A.; Cruguel, H.; Ouerghi, A.; Lacaze, E.; Silly, M. G.; Lhuillier, E. Probing Charge Carrier Dynamics to Unveil the Role of Surface Ligands in HgTe Narrow Band Gap Nanocrystals. J. Phys. Chem. C 2018, 122 (1), 859-865.

(28) Sadakane, M.; Steckhan, E. Electrochemical Properties of Polyoxometalates as Electrocatalysts. Chem. Rev. 1998, 98 (1), 219-238.

(29) Huder, L.; Rinfray, C.; Rouchon, D.; Benayad, A.; Baraket, M.; Izzet, G.; Lipp-Bregolin, F.; Lapertot, G.; Dubois, L.; Proust, A.; et al. Evidence for Charge Transfer at the Interface between Hybrid Phosphomolybdate and Epitaxial Graphene. Langmuir 2016, 32 (19), 4774-4783.

(30) Huang, J.; Liu, W.; Dolzhnikov, D. S.; Protesescu, L.; Kovalenko, M. V.; Koo, B.; Chattopadhyay, S.; Shenchenko, E. V.; Talapin, D. V. Surface Functionalization of Semiconductor and Oxide Nanocrystals with Small Inorganic Oxoanions (PO43-, MoO42-) and Polyoxometalate Ligands. ACS Nano 2014, 8 (9), 9388-9402.

(31) Yu, X.; Liu, R.; Zhang, G. Polyoxometalate-CdS Quantum Dots Co-Sensitized TiO2 Nanorods Array: Enhanced Charge Separation and Light to Electricity Conversion Efficiency. RSC Adv. 2013, 3 (22), 8351-8355.

(32) Chen, L.; Chen, W.; Tan, H.; Li, J.; Sang, X.; Wang, E. The Improved Efficiency of Quantum-DotSensitized Solar Cells with a Wide Spectrum and Pure Inorganic Donor-Acceptor Type Polyoxometalate as a Collaborative Cosensitizer. J. Mater. Chem. A 2016, 4 (11), 4125-4133.

(33) Rocchiccioli-Deltcheff, C.; Fournier, M.; Franck, R.; Thouvenot, R. Vibrational Investigations of Polyoxometalates. 2. Evidence for Anion-Anion Interactions in Molybdenum(VI) and Tungsten(VI) Compounds Related to the Keggin Structure. Inorg. Chem. 1983, 22 (2), 207-216. (34) Souchay, P. Polyanions and Polycations; Gauthier-Villars, Paris; 1963. 
(35) Nag, A.; Kovalenko, M. V.; Lee, J.-S.; Liu, W.; Spokoyny, B.; Talapin, D. V. Metal-Free Inorganic Ligands for Colloidal Nanocrystals: S2-, HS-, Se2-, HSe-, Te2-, HTe-, TeS32-, $\mathrm{OH}_{-}$, and NH2as Surface Ligands. J. Am. Chem. Soc. 2011, 133 (27), 10612-10620.

(36) Wang, C.; Shim, M.; Guyot-Sionnest, P. Electrochromic Nanocrystal Quantum Dots. Science 2001, 291 (5512), 2390-2392.

(37) Koh, W.; Koposov, A. Y.; Stewart, J. T.; Pal, B. N.; Robel, I.; Pietryga, J. M.; Klimov, V. I. Heavily Doped $\mathrm{N}$-Type PbSe and PbS Nanocrystals Using Ground-State Charge Transfer from Cobaltocene. Sci. Rep. 2013, 3, 2004.

(38) Volatron, F.; Noël, J.-M.; Rinfray, C.; Decorse, P.; Combellas, C.; Kanoufi, F.; Proust, A. Electron Transfer Properties of a Monolayer of Hybrid Polyoxometalates on Silicon. J. Mater. Chem. C 2015, 3 (24), 6266-6275.

(39) Ng, K. T.; Hercules, D. M. XPS Studies of Oxides of Row Transition Metals of W. J. Phys. Chem. C 1976, 80, 2095.

(40) McGuire, G. E.; Schweitzer, G. K.; Carlson, T. A. Core Electron Binding Energies in Some Group IIIA, VB, and VIB Compounds. Inorg. Chem. 1973, 12 (10), 2450-2453.

(41) Lhuillier, E.; Ithurria, S.; Descamps-Mandine, A.; Douillard, T.; Castaing, R.; Xu, X. Z.; Taberna, P.L.; Simon, P.; Aubin, H.; Dubertret, B. Investigating the N- and P-Type Electrolytic Charging of Colloidal Nanoplatelets. J. Phys. Chem. C 2015, 119 (38), 21795-21799.

(42) Sahu, A.; Kang, M. S.; Kompch, A.; Notthoff, C.; Wills, A. W.; Deng, D.; Winterer, M.; Frisbie, C. D.; Norris, D. J. Electronic Impurity Doping in CdSe Nanocrystals. Nano Lett. 2012, 12 (5), 25872594. 
TOC Graphic

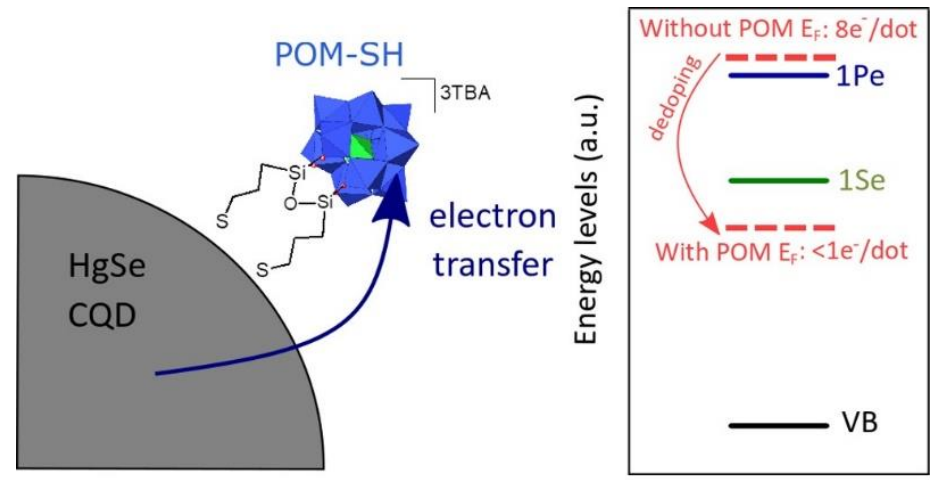

